\title{
Communication transculturelle et « lingua franca »
}

\author{
Françoise Buffet et Gérard Willems
}

\section{OpenEdition}

Journals

Édition électronique

URL : http://journals.openedition.org/ries/4043

DOI : $10.4000 /$ ries.4043

ISSN : 2261-4265

\section{Éditeur}

Centre international d'études pédagogiques

\section{Édition imprimée}

Date de publication : 1 juin 1995

Pagination : 61-72

ISSN : 1254-4590

\section{Référence électronique}

Françoise Buffet et Gérard Willems, "Communication transculturelle et « lingua franca » », Revue internationale d'éducation de Sèvres [En ligne], 06 | 1995, mis en ligne le 01 janvier 2015, consulté le 10 décembre 2020. URL : http://journals.openedition.org/ries/4043 ; DOI : https://doi.org/10.4000/ries. 4043

Ce document a été généré automatiquement le 10 décembre 2020.

(c) Tous droits réservés 


\title{
Communication transculturelle et « lingua franca »
}

\author{
Françoise Buffet et Gérard Willems
}

«An intercultural approch to the teaching of culture is radically different from a transfer of

information between cultures. It includes a reflection both on the target and on the native

culture. » (Kramsch, 1993)

[...] « Une approche interculturelle de l'enseignement de la culture diffère radicalement d'un transfert d'information entre cultures. Cette dernière implique une réflexion tant sur la civilisation cible que sur la civilisation native. » « The key to understanding language in context is to start not with the language but with the context. » (Hymes, 1972)

« La clé de la compréhension du langage contextuel est dans son approche : au départ il ne faut pas le langage, mais le contexte. »

Par tradition, l'enseignement des langues étrangères et la formation des professeurs se concentraient sur les aspects linguistiques de l'apprentissage de la langue. Le développement de la communication internationale et interculturelle engendré par une mobilité de plus en plus grande, à quoi viennent s'ajouter les signes d'une croissance globale de nationalismes et d'ethnocentrismes, nécessite une approche nouvelle de l'enseignement des langues. Une orientation plutôt morale/éthique, et par conséquent pédagogique, s'impose. Certes, cet appel s'adresse à l'ensemble du système éducatif, mais par leur nature même, c'est à l'enseignement et à l'apprentissage des langues étrangères de jouer un rôle essentiel dans l'internationalisation de l'éducation. L'expression la plus saisissante et la plus complexe de la culture se fait au moyen du langage. Ainsi, la prise de conscience de la différence culturelle, la volonté et l'aptitude à prendre en compte cette 
différence dans la communication interculturelle, constituent des domaines à développer prioritairement auprès des élèves et des étudiants.

Pour soutenir les professeurs de langues dans la mise en œuvre de cette tâche nouvelle et leur permettre de devenir ceux qui faciliteront la communication interculturelle, il faut élaborer de nouveaux supports pédagogiques et une méthodologie nouvelle d'autant que l'enseignement des langues étrangères cesse de concerner les seuls linguistes.

\section{Point de vue sur la formation à l'enseignement des langues étrangères}

\section{Finalité de l'enseignement des langues étrangères}

3 Depuis trop longtemps déjà, l'enseignement des langues vivantes et la formation des professeurs de langues étrangères sont dominés essentiellement par l'analyse grammaticale et l'apprentissage du vocabulaire, comme si l'apprentissage d'une langue étrangère ne devait pas s'étendre au-delà de l'appropriation d'un code différent ou de la répétition mécanique de phrases dans un livre de textes. Dans le meilleur des cas, les professeurs se sont contentés d'approuver pour la forme l'étude de la civilisation dans laquelle la langue est enracinée. Avec l'approche communicative, l'intérêt porté à l'étude de la civilisation accuse une tendance à la hausse. Cette dernière tend pourtant à se concentrer trop exclusivement sur l'information relative à la communauté ciblée, au lieu d'illustrer le fait que la compétence interculturelle représente un phénomène bien plus complexe que l'action de "faire des choses avec des paroles ». Ce qui a manqué dans ce type d'étude de la civilisation, c'était une invitation à voir les choses dans le prolongement de sa propre « logique » culturelle personnelle afin de développer ainsi un véritable savoir à propos de l'interprétation contextualisée, socialement et culturellement, des actes de parole. Comme l'écrit Byram (1992) :

Il est devenu d'usage d'enseigner les langues étrangères [...] comme si les professeurs devaient préparer leurs apprenants au rôle de touriste et de vacancier dans le pays étranger. [...] Ceci n'a pourtant aucun impact sur leur façon de voir leur identité propre, ni celle des autres ; ils sont implicitement sollicités de rester fermement ancrés dans leur propre système de valeurs et dans leur propre culture.

Les matériels pédagogiques existants n'invitent guère ou pas du tout les étudiants à acquérir un autre mode leur permettant de se définir eux-mêmes et d'avoir un accès social à un autre système de valeurs, d'attitudes et de goûts, puisque ces matériels ont pour seul effet de les conforter dans les valeurs, opinions, attitudes et goûts qui sont les leurs. Les matériels présentent presque toujours des locuteurs natifs exemplaires - des conversations enregistrées par des gens du pays, avec des messages positifs (pas de confidences, de remarques évasives, de double sens, etc.), dans des situations à caractère neutre (aéroports, concerts pop, gares de chemins de fer et autres) qui ne préparent pas l'étudiant à affronter des situations de la réalité courante, lorsque la lecture du contexte par les personnes participant à la communication peut être tout à fait différente.

De telles situations demandent que soient détectés les éventuels secteurs de malentendus. Elles présupposent un certain degré de connaissance à propos d'autres civilisations vidées de leurs stéréotypes. Les matériels en usage risquent de renforcer auprès de l'élève l'idée que ce qui compte en définitive n'est que sa propre façon de penser et de vivre, au lieu de l'aider à comprendre que sa propre culture, sa propre identité sociale ne représentent pas 
l'ultime mesure des choses du fait qu'elles lui sont familières. L'enseignement des langues étrangères devrait mettre l'élève en situation de remettre en question ses propres points de vue et d'engager ouvertement des négociations, avec l'autre et avec lui-même, afin de découvrir les significations éventuelles qui se cachent derrière ce qui se dit et se fait (Willems, 1991 ; Byram, 1992).

6 Tout cela demande une définition de la notion de civilisation et des facteurs complexes qui constituent la communication entre deux interlocuteurs qui partagent une (ou plusieurs) « lingua franca » (langues véhiculaires).

\section{Civilisation et communication en « lingua franca »}

7 La civilisation a été définie par un anthropologue culturel comme « la programmation collective de l'esprit qui différencie les membres d'un groupe ou d'une catégorie humaine d'un autre » (Hofstede, 1980, 1984, 1991). Sans être une définition complète, cela traduit ce que Hofstede est parvenu à mesurer. La civilisation est un savoir social, au sens le plus large du terme, que l'on acquiert en grandissant dans une certaine communauté par le biais de l'esprit qui est "programmé » dans le contact social. Elle ne relève pas d'un héritage biologique Nurture not Nature. Elle contient ce qu'il faut savoir afin de pouvoir fonctionner socialement et interpréter le monde de la même manière que ceux avec lesquels on vit.

8 Un tel savoir est composé, d'une part, d'un savoir à long terme, philosophies politiques et religieuses, théories sur les fonctionnements structurels d'une société et, d'autre part, un savoir à court terme, de nature plutôt éphémère, concernant les événements qui occupent les esprits d'un groupe de personnes à un moment donné. Elle comprend enfin un savoir-faire qui dit comment se comporter correctement, comment s'exprimer et interpréter adéquatement ce qui est dit et fait. L'acceptabilité est essentiellement déterminée par quatre dimensions : la façon dont les gens se situent dans un contexte mal connu, la façon dont ils se situent par rapport au pouvoir, leur degré d'intégration dans les groupes, et la différence entre les rôles sociaux de l'homme et de la femme (Hofstede, 1991).

\section{L'interaction dans une « lingua franca »}

Dès qu'il s'agit de communication, c'est le savoir culturel qui détermine la « lecture » du contexte. Il s'agit d'un savoir partagé et par conséquent d'une interprétation partagée par les participants dans un contexte facilitant la communication. C'est l'absence d'un tel savoir qui provoque des malentendus ou l'échec communicatif. Un savoir linguistique étendu combiné avec un savoir très limité de la civilisation cible ne nous mène pas aussi loin qu'un savoir linguistique restreint (mais approprié, cela va de soi) contextualisé par une expérience vécue de la communauté de la langue cible.

Il s'agit là d'un raisonnement généralement avancé pour des personnes qui communiquent dans une langue étrangère avec une personne dont c'est la langue maternelle. La situation change un peu, quand on analyse la communication entre deux interlocuteurs utilisant une langue qui est étrangère à l'un et l'autre. En fait, dans une interaction de la sorte, trois « civilisations » sont impliquées. La civilisation d'origine de chacun des participants et celle de la langue véhiculaire utilisée. Souvent, les irritations consécutives aux malentendus risquent d'entraver fâcheusement ce genre de 
communication et elles ne contribuent pas à développer des attitudes positives entre les interlocuteurs.

11 Ici nous abordons un sujet extrêmement délicat. On constate que le locuteur natif est devenu ces derniers temps une espèce dont on parle beaucoup. Il existe des nations qui ont le sentiment, souvent pour des raisons d'ordre historique, que leur civilisation d'origine les empêche d'adopter le comportement requis par la civilisation de la lingua franca utilisée. Ainsi, nombreux sont les Allemands qui ont le sentiment que les circonlocutions des Britanniques ne correspondent pas à leur comportement habituel. Ils ont du mal à s'exprimer par exemple dans les termes suivants : «I am afraid I do not quite see eye to eye with you in this matter » pour dire : «Das stimmt aber nicht. - That's wrong - Vous vous trompez " (Beneke, 1993). Or, la question est de savoir dans quelle mesure, dans l'enseignement des langues, il est nécessaire d'insister sur un modèle de l'usage social d'une lingua franca, et si cela est acceptable.

Pour des raisons évidentes, nous acceptons depuis longtemps une prononciation et un modèle grammatical standard de la langue cible. Les choses seraient plus simples si les usages de la langue véhiculaire possédaient les fondements de la culture de cette langue : cela faciliterait la communication et l'interaction. Mais ce n'est pas souvent le cas, et le locuteur natif est induit en erreur lorsqu'il entend parler sa langue avec un accent et une grammaire corrects : $i$ suppose que l'interlocuteur a les mêmes références culturelles que lui. À cet égard, les communications en langue étrangère et en lingua franca sont comparables : les participants, y compris le locuteur natif de la langue utilisée, devront tenir compte de "trous » culturels dans la compétence linguistique de chacun (i.e. de la langue non maternelle). Si le locuteur natif a lui-même l'expérience de l'apprentissage d'une langue étrangère, il sera d'autant plus apte à percevoir les difficultés lorsqu'elles se présentent.

\section{Dimension internationale et médiation culturelle dans l'enseignement des langues étrangères}

Les questions mises en avant par l'usage de la lingua franca s'inscrivent dans un changement des paradigmes sociologiques de la connaissance. Depuis le $\mathrm{xvI}^{\mathrm{e}}$ siècle, le paradigme dominant a été celui de l'individualisation et de la séparation. Depuis les années 1980, au contraire, la globalisation s'impose : l'informatique et l'évolution des télécommunications sont des éléments majeurs de ce changement. On part du global pour aller vers le particulier afin d'y retrouver le spécifique.

Dans le même temps, les systèmes éducatifs européens ont été marqués par l'importance croissante des migrations. Par force, on voit émerger une nouvelle problématique de l'enseignement des langues, précoce ou non. Cette problématique est révélatrice de la volonté d'adaptation à un changement de société. La mobilité accrue des individus, tant géographique que sociale, favorise la confrontation culturo-linguistique qui cesse d'être le fait d'une microsociété internationale. La communication, phénomène de masse alimenté par des technologies nouvelles, a lieu en temps réel entre les différents partenaires et le dialogue s'inscrit dans l'immédiateté de l'action.

Comme les enjeux sont différents, les buts de l'enseignement changent. La compréhension prime sur l'expression, au moins dans un premier temps. Lorsqu'on ne vise plus la formation de spécialistes en langue, la tolérance quant à l'usage académique 
de la langue cible est plus large. Plus exactement, le réfèrent culturel cesse d'être un réfèrent scolaire et littéraire unique pour devenir un réfèrent pluriel qui tient compte de la variété des cultures et des langages, en rapport avec la variété des groupes sociaux. On reconnaît la validité provisoire de constructions grammaticales intermédiaires, mais cohérentes, dès lors qu'elles favorisent le développement des idées, de la créativité et de la communication. Cette stratégie de la tolérance, ce droit à l'erreur, donne la priorité à la pratique de formulation personnelle (L. Vallès Ferrer, 1989). Cela répond à l'objectif de maitrise d'habiletés communicationnelles par le plus grand nombre.

De ce fait la problématique change : on passe de l'objectif d'apprentissage "académique " d'une langue étrangère, à un objectif de " communication nécessaire et suffisante » pour le plus grand nombre. Nécessaire pour développer chez l'étudiant un processus cognitif d'interrogation, d'information, d'acquisition et de résolution des problèmes ordinaires d'une communication interculturelle. Suffisante pour participer selon des modalités démocratiques aux processus de construction de la société.

Pour cela, chacun doit pouvoir participer à la dynamique culturelle qui met en jeu à la fois la réalité des cultures concernées avec leur potentiel de développement et les virtuels issus des confrontations, des consensus et des compromis qui ont lieu entre les acteurs en présence. Le résultat de cette dynamique est la médiation culturelle. Par médiation culturelle, on entend l'ensemble des interactions et mises en synergies qui reconstituent, à partir des éléments de plusieurs cultures, des schèmes transversaux, en partie communs aux différents partenaires. Cette médiation permet de passer de l'imaginaire au symbolique et de l'individuel au collectif. Ce processus correspond à un changement dans les mentalités : rupture avec le cadre de la modernité, acceptation de l'aléatoire comme un élément « normal » de la dynamique sociale et conjonction entre une morale sociale et une éthique du sujet responsable, autonome, et solidaire. Mais, paradoxalement, alors que la différenciation culturelle tend à se réduire, l'affirmation des identités s'accroît: les dynamiques d'organisation l'emportent sur les structures, le jeu de l'acteur dans le système devient déterminant et l'éthique du sujet prend le pas sur la morale sociale.

Un tel point de vue est en rupture avec les pratiques actuelles de formation des enseignants qui ignorent les processus de médiation et la formation au partenariat culturel éducatif.

\section{La perception culturelle fondement de la médiation culturelle}

La communication entre les individus repose sur des stratégies (prise de parole, explications, argumentations, etc.) qui sont liées à la perception intuitive et spontanée des différences culturelles (Willems, 1993a). Dans la volonté de communiquer, il y a la volonté de se faire comprendre, d'établir un «chiffre " commun, donc il y a nécessité d'ajuster son discours pour qu'il soit « recevable » par le destinataire. La construction du chiffre commun commence avec l'accord sur la signification première du vocabulaire, mais elle relève d'un processus plus large de compréhension; elle peut être analysée à différents niveaux de complexité et elle peut être saisie d'abord dans la société et la culture d'appartenance avant d'être transposée à la relation entre des cultures dites étrangères.

Deux exemples illustrent ceci. Le premier est celui d'une discussion entre formateurs anglais, français et néerlandais dans laquelle le terme "didactique» était le mot-clé. Visiblement, il y avait une incompréhension qui se traduisait par une attitude de réserve 
courtoise du collègue britannique jusqu'au moment où il est apparu que didactic désignait en anglais un mode d'enseignement que l'on qualifie d'académique (transmissif) en français. En fait, à l'arrière-plan, c'était un conflit idéologique sur la démarche d'enseignement et la relation à l'élève qui se dessinait. Le second exemple est celui d'une discussion entre étudiants danois et grecs sur la question de l'utilisation de l'eau lors de la préparation d'une leçon pour l'école primaire. Le choc des cultures fut rude! Ici, c'est plus une confrontation de systèmes de valeurs qui est mise en évidence. Dans un cas comme dans l'autre, il est évident que la construction du "chiffre commun » dépend d'abord de l'identification de ses propres valeurs culturelles, sociales et personnelles et de celles des partenaires afin d'établir des équivalences partielles entre les différents systèmes. Cette construction suppose un double effort de décentration et d'universalisation, pour amorcer le processus de médiation culturelle.

21 Si les partenaires ont réellement la volonté de communiquer, la perception des différences culturelles déclenche une attitude positive d'« éveil » à l'autre : si le " groupe culturel» de mon interlocuteur m'est étranger, je suis sensible aux différences de "tournures " et j'ai une incertitude permanente sur le sens et les nuances des mots employés car c'est la contextualisation culturelle et événementielle qui donne le sens au discours. L'incertitude me condamne à établir une "table d'équivalence", donc à chercher des éléments de référence autant, sinon plus, dans la façon d'être de mon interlocuteur et dans la valeur symbolique accordée aux objets, aux fonctions et aux rôles, que dans le vocabulaire ou dans la construction des phrases.

La perception de la différence culturelle est donc plus ou moins fine selon l'individu, sa culture, l'objet plus ou moins concret de la conversation, le contexte même de l'échange, et la perception plus ou moins élaborée de ce qu'est une communication interculturelle. Elle se fait au moyen de schèmes, sortes de grilles de lecture, qui permettent de décoder les constructions linguistiques et qui opèrent dans la dynamique de l'interaction, permettant la construction des référents communs, à partir de l'identification des éléments qui peuvent supporter le plus facilement des modifications (ou sur lesquels on pense que le partenaire acceptera de transiger). Le fait de communiquer, avec les moyens dont on dispose, amorce l'interaction qui fait que l'on se sent «capable» de communiquer, et la première compétence de communication naît dans la pratique de la médiation culturelle.

23 La médiation, fondamentale car elle commande l'intégration de la dimension internationale dans la " mentalité », se fait dans et par une culture virtuelle de référence, sorte de tiers-culturel résultant de la combinaison des habitus (P. Bourdieu, La théorie de la pratique) culturels des locuteurs, des potentiels des cultures de référence et, éventuellement, de la colonisation de la culture de la lingua franca par ceux qui l'utilisent (ce qui est le cas dès que l'on aborde les langues de faible diffusion).

24 Le tiers-culturel, résultat du processus de médiation culturelle, permet de passer de la dialectique ressemblance-différence à une nouvelle dialectique ressemblance-différenceéquivalence car il contient et fait fonctionner les systèmes de valeurs connus et reconnus par les uns et les autres, souvent différents mais tolérés, et qui correspondent à une éthique du sujet. Si on considère les différentes disciplines d'enseignement, on constate la carence de formation systématique en langues et en sociologie des futurs enseignants du primaire et du secondaire, toutes disciplines confondues. En développant la capacité d'analyse des codes sémiotiques, fondement de la médiation, ces disciplines facilitent la mise en équivalence des systèmes de valeurs, des attitudes et des affects, des 
comportements sociaux. Favorisant la reconnaissance culturelle entre les partenaires, elles sont un élément important du travail sur les mentalités en vue de la prise en compte de la dimension internationale.

\section{Compétence interculturelle, contexte social et implications didactiques}

\section{Des fondements de la compétence stratégique de communication interculturelle...}

Pour essayer de réaliser les ambitions pédagogiques de l'enseignement et de l'apprentissage des langues étrangères, on prendra en compte les considérations suivantes qui fondent la compétence interculturelle. Cette compétence est basée sur :

- les caractéristiques personnelles: ouverture aux contacts sociaux ou le contraire; orientation sur l'harmonie ou sur le conflit ;

- l'aptitude communicative : la gamme des aptitudes linguistique, pragmatique et discursive d'une personne, ce qu'elle sait et ce qu'elle sait faire, sa perception des métacommunications ;

- la volonté de communiquer vraiment avec les représentants d'autres civilisations: la conviction que la civilisation est une donnée relative qui échappe à une hiérarchisation mettant sa propre culture au premier rang;

- le savoir socioculturel qui permet à la fois d'avoir conscience de sa propre culture et des relations qu'elle entretient avec d'autres cultures et, en même temps, de relativiser et transcender cet état de fait.

Si l'on pouvait réunir toutes ces conditions dans l'enseignement scolaire, cela simplifierait les choses. Il faudra par conséquent faire appel à l'enseignement des langues pour aider l'étudiant à développer ses compétences communicatives dans une perspective de prise de conscience culturelle du contexte social.

\section{... à la stratégie de formation, la dimension internationale}

Étant donné que tout usage d'une langue entre gens raisonnables est finalisé, le comportement stratégique devra être au centre de la compétence communicative. La compétence stratégique est liée à la civilisation et est en droit d'occuper, en conséquence, une place centrale quand il s'agit de compétence communicative. L'enseignement des langues étrangères devrait viser à sensibiliser l'étudiant à la différence culturelle en mettant l'accent sur la gamme de stratégies dont on peut se servir dans la communication (Willems, 1993a, b). Il faudrait analyser et étudier des exemples d'interactions entre personnes utilisant leur langue maternelle pour les comparer à l'interaction entre locuteurs non natifs et à celle des locuteurs natifs et des locuteurs non natifs dans des situations similaires. Des exercices à trous invitant à compléter une information, une opinion, une émotion se prêtent à clarifier ces interactions (Willems, 1995).

Kramsch (1993), qui prétend que toute classe de langue, même celle des personnes ayant une langue maternelle commune, est interculturelle, décrit les discussions menées dans une classe de langue portant sur l'interprétation d'un poème. Ainsi, les étudiants sont encouragés à appréhender le rapport complexe liant langue et culture. C'est le début 
d'une prise de conscience de la différence entre deux civilisations. Cela les entraînera à créer une troisième perspective permettant de situer ces différences pour en discuter sans crainte. Dans ce processus, ils se rendront compte simultanément que leur logique culturelle propre est une logique parmi d'autres, ni supérieure ni inférieure.

De façon complémentaire, l'expérience vécue au sein des Réseaux d'instituts de formation, regroupant des formateurs de langues, mais aussi des géographes et des sociologues, a permis de prendre conscience du poids de l'approche normative caractéristique de tout travail direct sur des phénomènes culturels pris comme " objet " d'enseignement/apprentissage. La première conclusion des travaux a été que les démarches de formation doivent d'abord mettre les étudiants en situation de prendre conscience des codes qui leur sont familiers, ce qui conduit à définir un dispositif didactique articulé autour de quatre axes transversaux par rapport aux domaines des disciplines :

- une éducation à la perception des codes culturels ;

- une analyse des perceptions et des représentations ;

- une analyse de la symbolique des objets qui fait apparaître, au-delà du choix de l'individu, ce qui étonne, ce qui est indispensable, ce qui est jugé significatif ;

- une production de nouveaux axes de représentation, par l'action: il s'agit de créer des situations de nécessité, dans lesquelles des sujets de cultures différentes doivent réaliser une action commune.

Plus concrètement, cela signifie que les étudiants doivent, dans un premier temps, être confrontés à une série de tâches qui les amènent à comparer leurs propres habitudes culturelles et celles des locuteurs de la langue cible, en fonction de contextes définis et différents. La réalisation de ces tâches doit être étalée sur une longue période (six mois environ) avant que les étudiants partent en stage à l'étranger. Les contextes des activités mises en place doivent être choisis et construits en partenariat avec les professeurs de la culture cible. Les travaux peuvent se présenter sous la forme de courtes séquences mettant en jeu l'expression d'informations, d'opinions ou d'émotions, qui placent les étudiants dans des situations les plus proches possibles de la réalité. Les étudiants réalisent ces exercices dans un premier temps entre eux, dans leur institut, et dans leur langue maternelle, puis dans la langue cible. Ces réalisations sont transcrites par les étudiants et contrôlées par le formateur.

31 Puis dans un deuxième temps, ils écoutent un enregistrement de la même situation fait par deux locuteurs natifs et comparent leurs stratégies réciproques. Enfin, ils répètent l'exercice et comparent leurs deux réalisations en langue étrangère en repérant les différences et en les analysants. Cette démarche est décrite par Willems (1994).

Il est très important de discuter avec les étudiants de l'existence des modèles culturels et de leurs constituants: schémas des propositions (qui spécifient la construction des concepts et les relations entre ceux-ci) et schémas des images (gestalts) tels qu'ils sont proposés par Holland et Quinn $(1987,1991)$. Ceci peut se faire sur la base des expériences des étudiants, par les exercices décrits ci-dessus. Au cours de ces activités les étudiants se rendent compte de la complexité de l'organisation du savoir social dans les modèles culturels.

La démarche suivante est l'étude des "stratégies de compensation" comme élément facilitateur de la communication transculturelle. Par exemple, en cas de doute, on dira: «Je vous entends dire que... Est-ce que cela signifie que... ? Ai-je mal compris ? Pour moi 
cela veut dire... ». En fait, la volonté d'acquérir les stratégies de compensation au cours de la communication avec les représentants d'une autre culture est essentielle. C'est le point de départ de la médiation culturelle qui fonde la mise en place du «chiffre commun » dont on a parlé. La négociation du savoir social, et des modèles culturels dans lesquels elle prend racine, est impossible sans cette stratégie de compensation.

Les étudiants réaliseront progressivement que l'acculturation est souhaitable, mais qu'elle est très difficile à atteindre, même en séjournant longtemps dans le pays étranger choisi. À condition de le préparer soigneusement, un tel stage est toutefois indispensable pour les étudiants en langues étrangères. Il leur offre la possibilité de mettre en œuvre les stratégies de compensation, et de produire un chiffre commun, clé de la construction culturelle ultérieure avec le partenaire étranger. C'est le début de la prise de conscience relative à l'importance de la coopération dans le processus de globalisation des civilisations auquel on assiste actuellement. L'éducation européenne s'inscrit dans ce processus d'internationalisation: ses propres perspectifs culturels sont relativisés et celles des autres sont considérées comme intrinsèquement intéressantes.

Parce qu'il est au cœur du débat, le cas de l'enseignement et de l'apprentissage des langues étrangères est significatif de l'effort à faire pour qu'évoluent les mentalités. Il existe toujours une tentation de dichotomie entre la technique propre au domaine disciplinaire, et la prise en compte dynamique du fait culturel.

Les professeurs de langue, c'est là l'essentiel, devraient bousculer les idées toutes faites de leurs étudiants à propos des civilisations étrangères. Les futurs enseignants de langues doivent apprendre à créer des expériences interculturelles dans leurs classes, afin d'ouvrir les esprits aux équivalences qui existent entre les cultures, afin aussi que les différences soient discutées dans une perspective de relativisation et de respect réciproque. La formation doit prendre en compte la volonté de médiation qui s'inscrit dans une problématique de la participation. Cela suppose un travail sur les légitimités, les réciprocités, les déséquilibres et les équivalences qui sont au cœur des systèmes d'action concrets afin d'établir une meilleure communication entre les citoyens du monde.

\section{BIBLIOGRAPHIE}

BENEKE J., « English as the Medium of Intercultural Communication: some Teaching Suggestions ", in: BEZNEKE J. (coord.), Communication in aviation, Bonn, Dümmler Verlag, 1993.

BERNOUX Philippe, La sociologie des organisations, Paris, Seuil, 1985.

BOURDIEU Pierre, Raisons pratiques, Paris, Seuil, 1994.

BUFFET Françoise, «Quelques questions pour les enseignants », in : IRDP/DELVAL, J.-A. TSCHOUMY (coord.), Vers une reconnaissance des échanges, Neuchâtel, 1992.

BUFFET Françoise., « La démarche de professionnalisation », in : Technologiel Educationale Moderne, publication du laboratoire Bazele technologeiei si mâiestrlei pedagogice, sous la direction du professeur MANDACANU, Chislnâu, Moldavie, 1994. 
BUFFET Françoise, « Formation au partenariat culturel et éducatif : enjeux et évaluations », in : Actes du colloque international des VII entretiens du Centre Jacques Cartier, Lyon, 1994.

BYRAM M., « Foreign Language Teaching for European Citizenship », in: Language Learning Journal , septembre 1992.

BYRAM M., MORGAN C, et al., Teaching-and-Learning Language-and-Culture, Clevedon, Multilingual Matters, 1994.

CLANET Claude, L'interculturel : introduction aux approches interculturelles en éducation et en sciences humaines, Toulouse, Presses universitaires du Mirail, 1990.

DAMISCH Hubert, Ruptures cultures, Paris, Éd. de Minuit, 1991.

DEMORGON Jacques, L'exploration interculturelle. Pour une pédagogie internationale, Paris, Colin, 1989 HABERMAS Jurgen, Théorie de l'agir communicationnel, Paris, Fayard, 1981/1987.

HALL E.T., Beyond Culture, New York, Anchor Press \& Doubleday, 1976.

HAROUEL Jean-Louis, Cultures et contre-cultures, Paris, Presses universitaires de France, 1994.

HOLLAND D., QUINN N (coord.), Cultural Model in Language and Thought, Cambridge, Cambridge University Press, 1987.

HOFSTEDE G., Culture's Consequences, Newbury Park CA, Sage Publications, 1980/1984.

HOFSTEDE G., Cultures and Organisations; Software of the Mind, London, Mc Graw Hill, 1991.

HYMES D., « Introduction » to: Cozden, John Hymes: Functions of Language in the Classroom, New York, Teacher's College Press, 1972.

KRAMSCH C, Context and Culture in Language Teaching, Oxford, OUP, 1993.

LADMIRAL Jean-René, LIPIANSKY Edmond MARC, La communication interculturelle, Paris, Colin, 1989.

RILEY Philip, « Social Identity and Intercultural Communication », Levende Talen, $\mathrm{n}^{\circ} 443$, septembre 1989.

SCHNAPPER Dominique, La France de l'intégration, Paris, Gallimard, 1991.

SERRES Michel, Le tiers instruit, Paris, éd. F. Bourin, 1991.

TOURAINE Alain, La production de la société, Paris, Seuil, 1983.

TOURAINE Alain, Critique de la Modernité, Paris, Fayard, 1993.

TOURAINE Alain, Qu'est-ce que la démocratie ?, Paris, Fayard, 1994.

VALLES FERRER L., «L'expression libre pour l'acquisition des langues », European Journal of Teacher Education, vol. 12, n² 1989.

WILLEMS G.M., " Foreign Language Use and the Growth of European Citizenship », Curriculum, vol. 12/3, p. 43-149, 1991.

WILLEMS G.M., " Oral Foreign Language Use: an Exercise in Cross-cultural Strategic Behaviour », in: BENEKE J. (coord.), Communication in aviation, Bonn, Dümmler Verlag, 1993a.

WILLEMS G.M., « Attainment Targets for Foreign Language Teacher Education in Europe; a European View », ATEE, cahier n 5, Brussels, 1993b.

WILLEMS G.M., « Foreign Language Conversational Skills in Tertiary Education: a Socio-cultural and Autonomous Approach », Language, Culture and Curriculum, vol. 7/3, 1994. 
OCDE, Redéfinir le curriculum : un enseignement pour le XXI siècle, Paris, OCDE, 1994.

\section{RÉSUMÉS}

Le développement de la communication internationale et l'usage fréquent d'une langue médiatrice, étrangère aux deux interlocuteurs, nécessitent une approche nouvelle de l'enseignement des langues qui tend à développer prioritairement l'aptitude à prendre en compte la différence culturelle dans la communication. Elle passe par une pédagogie de la médiation culturelle où se construit le "tiers culturel ", c'est-à-dire les systèmes de valeurs connus et reconnus, souvent différents mais tolérés par les uns et les autres.

INDEX

Index géographique : France

Mots-clés : coopération internationale, dimension européenne, échange international, enseignement des langues, formation des enseignants, pluralisme culturel

\section{AUTEURS}

\section{FRANÇOISE BUFFET}

Maître de conférences, IUFM de Lyon, Centre de Bourg-en-Bresse, France.

\section{GÉRARD WILLEMS}

Responsable du Département d'anglais, Center for International Education, Nimègue, Pays-Bas. 